\title{
A new elasto-plastic spring element for cyclic loading of piles using the p-y curve concept
}

\section{Hededal, Ole; Klinkvort, Rasmus Tofte}

Published in:

Numerical Methods in Geotechnical Engineering

Publication date:

2010

Document Version

Publisher's PDF, also known as Version of record

Link back to DTU Orbit

Citation (APA):

Hededal, O., \& Klinkvort, R. T. (2010). A new elasto-plastic spring element for cyclic loading of piles using the py curve concept. In Numerical Methods in Geotechnical Engineering: NUMGE 2010 (1 ed., Vol. 1, pp. 883-888). Taylor and Francis Group.

\section{General rights}

Copyright and moral rights for the publications made accessible in the public portal are retained by the authors and/or other copyright owners and it is a condition of accessing publications that users recognise and abide by the legal requirements associated with these rights.

- Users may download and print one copy of any publication from the public portal for the purpose of private study or research.

- You may not further distribute the material or use it for any profit-making activity or commercial gain

- You may freely distribute the URL identifying the publication in the public portal 
Offshore geotechnical engineering 


\title{
A new elasto-plastic spring element for cyclic loading of piles using the p-y-curve concept
}

\author{
Ole Hededal \& Rasmus Klinkvort \\ Department of Civil Engineering, Technical University of Denmark
}

\begin{abstract}
Modeling the response of large diameter piles subjected to lateral loading is most often done by means of $\mathrm{p}$-y-curves in combination with Winkler beam models. Traditionally the $\mathrm{p}$-y curves are formulated as non-linear (elastic) relations between the lateral movement $y$ and the soil response pressure $p$ in terms of monotonic loading (until failure) as e.g. prescribed by API (2000). However, the cyclic and dynamic performance is only to a limited degree accounted for. Here the elasto-plastic framework is applied allowing definition of unloading-reloading branches, hence enabling modeling of cyclic response. The present model can account for effects like pre-consolidation and creation of gaps between pile and soil at reversed loading. Results indicate that the model is able to capture hysteresis during loading with full cycles and model the accumulated displacement observed on piles subjected to "half cycles" as e.g. seen from centrifuge tests carried out. This article presents the theoretical formulations, discusses numerical implementation and finally presents simulations.
\end{abstract}

\section{INTRODUCTION}

Modeling the response of large diameter piles subjected to lateral loading is most often done by means of p-y-curves in combination with Winkler beam models. Traditionally, the p-y curves are formulated in terms of non-linear (elastic) relations between the lateral movement $y$ and the soil response pressure $p$ in terms of monotonic loading (until failure). These curves were established by back-analysis of a series of tests carried out in the $1950^{e s}$ by Matlock and co-workers. The tests were primarily static, monotonic load tests, but also a few cyclic tests were carried out.

Matlock (1970) carried out further cyclic tests on piles in clay that revealed a general reduction of the ultimate capacity for piles subjected cyclic loading compared to monotonic loading. This led to a general reduction of the cyclic ultimate capacity compared to the monotonic ultimate capacity. This reduction or cyclic degradation as it is commonly denoted is incorporated in almost all design codes, e.g. API (2000), as a formal reduction of the ultimate capacity. Still, the models does not directly correlate the reduction to the characteristics of the cyclic loading, i.e. number of cycles, loading amplitude or frequency.

Matlock (1970) and later Mayoral et al. (2005) set up a conceptual model for pile-soil interaction from these observations, cf. Figure 1. The model consists of 3 parts. Firstly, a loading phase where the soilpile interaction follows the virgin curve. Secondly, an unloading phase that due to irreversible deformations in the soil will imply the development of a gap between the pile and the soil. Finally, a phase where the pile moves towards the initial position and into the opposite

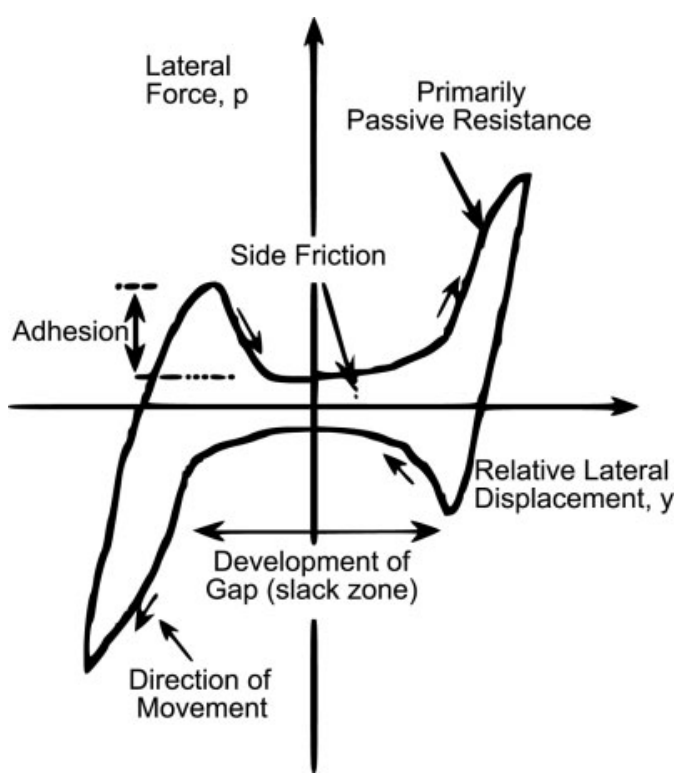

Figure 1. Typical loading cyclic for a model pile in clay, from Mayoral et al. (2005).

soil face in the cavity created behind the pile during initial loading. In this phase it may be assumed that there exists a drag or friction along the side of piles. Whether or not the gap will develop may depend on the type of soil type. El-Naggar et al. (2005) assumes that the gap will develop for cohesive soils, whereas for cohesionless soils, the soil will cave in and close the gap. Still, centrifuge tests carried out on a pile in 
dry sand indicate that this cave-in effect may not be fully developed, Klinkvort (2009), thus there is probably a need to include the drag effect in a model even for cohesionless soils. Klinkvort (2009).

One of the first attempts in formulating $\mathrm{p}-\mathrm{y}$-curves that reflected the observed behavior was done by Matlock et al. (1978). Later, Boulanger et al. (1999) proposed an elasto-plastic p-y model based on a two component set-up in which the loading response is handled by a series connection of springs - one spring handling loading (passive failure mode) and another spring handling the unloading-reloading properties of a pile subjected to cyclic loading that is gradually creating a gap behind the pile. Taciroglu et al. (2006) further developed these ideas and proposed a macroelement consisting of three components; leading-face element, rear-face element and drag-element. The two face-elements are formulated in terms of elasto-plastic springs supplemented with a tension cut-off. The drag element controls the side friction, when the pile is moving inside the cavity during unloading.

In the present work, the principles of the abovementioned models are incorporated in a single spring element that can be directly incorporated in a standard finite element code. In the following the elasto-plastic constitutive relations will be presented. Then follows a discussion about the implementation and finally some results from simulations.

\section{ELASTO-PLASTIC MODEL}

A simple one-dimensional elasto-plastic spring is defined. The model is expressed in terms of the earth resistant force $p$ and the associated displacement $u$.

The standard procedure for development of elastoplastic models are used. First the operator split between elastic and plastic components is assumed.

$d u=d u^{e}+d u^{p}$

where $d u^{e}$ is the elastic part and $d u^{p}$ is the plastic part of the total displacement increment $d u$.

The plastic displacement component is defined in terms of the gradient to the plastic potential, i.e.

$d u^{p}=d \lambda \frac{\partial g}{\partial p}$

with $d \lambda$ as the plastic multiplier. The direction of the plastic displacement increment is fixed to the loading plane, implying that the plastic flow potential is by definition associated to the yield surface, i.e. $f=g$.

The simplest yield function may be written as

$f=|p|-p_{u}(\boldsymbol{\alpha})=0$

in which $p_{u}(\boldsymbol{\alpha})$ is the current strength yield strength and $\boldsymbol{\alpha}=\left(\alpha_{1}, \alpha_{2}, \ldots\right)$ are the hardening parameters (to be defined later).
As mentioned above the flow rule is associated to the yield function, hence rewriting Eqn. (2) by use of Eqn. (3), we find

$d u^{p}=d \lambda \frac{\partial f}{\partial p}=\frac{p}{|p|} d \lambda$

In case of plastic loading $f=0$ the consistency requirement requires the stress point to remain on the yield surface, hence

$d f=\frac{\partial f}{\partial p} d p+\frac{\partial f}{\partial \boldsymbol{\alpha}} \frac{\partial \boldsymbol{\alpha}}{\partial \lambda} d \lambda=\frac{p}{|p|} d p-H d \lambda=0$

where the hardening modulus $H$ is the scalar contraction of the partial derivatives of the yield function with respect to $\boldsymbol{\alpha}$. For isotropic hardening, only a single hardening parameter is needed, i.e. $\boldsymbol{\alpha} \equiv \alpha$, but since we need to account for the development of a gap on the front and on the rear of the pile, respectively, it is necessary to introduce two hardening parameters as is presented in the coming sections.

As always the fundamental assumption of common elastic and plastic stress is used, hence

$d p=k d u^{e}=k\left(d u-d u^{p}\right)=k\left(d u-\frac{p}{|p|} d \lambda\right)$

where $k$ is the elastic stiffness. Combining Eqn. (5) and Eqn. (6) yields the definition of the plastic multiplier $d \lambda$,

$d \lambda=\frac{p}{|p|} \frac{k}{k+H} d u=\frac{k}{k+H}|d u|$

Here it is used that the displacement increment is associated to the loading direction, hence $p \cdot d u=1$.

This relation is then entered back into Eqn. (6) to produce the elasto-plastic tangent stiffness,

$k^{e p}=\frac{k H}{k+H}$

This completes the formal definition of the plasticity model. Remaining is now to define the yield strength as a function of the hardening parameters.

\subsection{Yield function}

Following the terminology of Mayoral et al. (2005) and Matlock (1970) we divide the current yield strength into two parts; one relating to the drag contribution and one relating to the earth pressure.

$p_{u}(\boldsymbol{\alpha})=p_{u}^{\mathrm{drag}}+p_{u}^{\mathrm{face}}(\boldsymbol{\alpha})$

The first term $p_{u}^{\mathrm{drag}}$ is the drag capacity, which in this version of the model is assumed to be constant. Below this value, the spring is assumed linear elastic with a stiffness $k$. The second term must account for the 


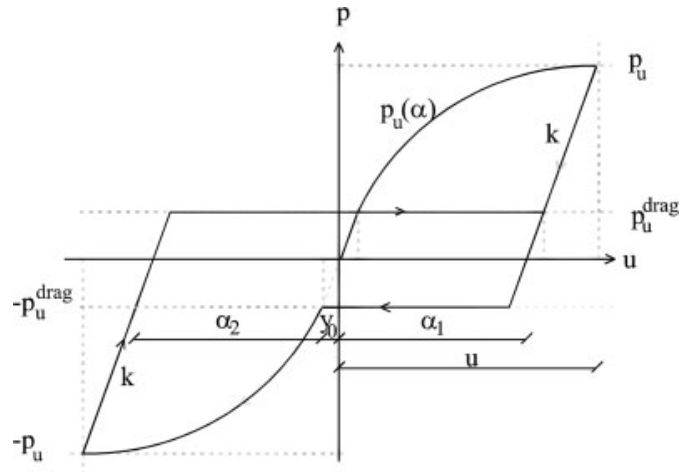

Figure 2. Schematic drawing of the spring element.

earth pressure when either of the pile faces are in contact with the soil. If there is no contact, this term must vanish. This can be achieved by introducing a multiplier to the virgin curve. The obvious candidate is a smooth step function,

$S(x)=\frac{1}{1+e^{-2 \beta x}}$

The parameter $\beta$ defines the curvature and the coordinate $x$ is

$x_{1}=u-p_{u}^{\mathrm{drag}} / k-\alpha_{1} \quad$ for $\quad u>0$

$x_{2}=-u+p_{u}^{\mathrm{drag}} / k+\alpha_{2}$ for $u<0$

A typical value for $\beta$ would be around 1.000 .000 . The coordinate $x$ thus defines the current position of the pile relative to the soil. If the pile is in contact with the soil $x \geq 0$ and if there is a gap $x<0$. Using Eqn. (9) we can write the yield function as

$p_{u}^{\mathrm{face}}(\boldsymbol{\alpha})=\sum_{i} S\left(x_{i}\right) p_{u}^{\mathrm{virgin}}\left(\alpha_{i}\right)$

The hardening parameters $\alpha_{i}, i=1,2$ represents either loading of the front or rear face of the pile. The virgin curve $p_{u}^{\text {virgin }}(\alpha)$ depends on the soil conditions as e.g. given by API (2000).

\subsection{Evolution law for hardening parameters}

Referring to Figure 2 it is easily seen that the hardening parameter $\alpha_{i}$ is defined as the plastic displacement accumulated during contact between soil and pile. Physically $\alpha$ is representing the progressive development of the gap. Using the experience from contact mechanics, it is deemed that a formulation of unloading and reloading in terms of a displacement criterion (rather than the usual stress based criterion) allows us to keep the formulation simple, even for the discontinuous phase when the pile is moving in the developed cavity.

The evolution law for the hardening parameters should thus be defined in such a way that they only develop when the pile is in contact with the soil. As long as the pile is sliding in the cavity created by the cyclic motion, the model should behave ideally plastic. Introducing once again the step function we may find

$d \alpha_{i}=S\left(x_{i}\right) d u^{p}=\frac{p}{|p|} S\left(x_{i}\right) d \lambda$

in which the definition of the plastic displacement, Eqn. (4), is utilized. Having established the evolution law, it is finally possible to identify the model specific hardening modulus, $H$, by revisiting the consistency equation, Eqn. (5). After some manipulation we find that

$$
\frac{p}{|p|} d p+\underbrace{\frac{p}{|p|} \sum_{i}\left[\frac{\partial p_{u}^{\text {virgin }}}{\partial \alpha_{i}} S^{2}+p_{u}^{\text {virgin }} \frac{\partial S}{\partial \alpha_{i}} S\right]}_{-H} d \lambda=0
$$

Note that the arguments $\alpha_{i}$ and $x_{i}$ has been omitted in the formula. Analyzing Eqn. (14), it is noted half of the contributions vanishes if the soil is in contact with either the front face or the rear face of the pile, since the for the unloaded face $S=0$. Likewise this relation ensures that $H=0$ in the cavity since $S=0$ for all terms.

\section{IMPLEMENTATION}

The proposed spring element is implemented in an inhouse MATLAB based FE code, Hededal and Krenk (1995). The implementation consists of two parts. Firstly implementation of the spring element using a backward Euler integration scheme for integration of the constitutive relation. Secondly, a Winkler model based on the proposed model has been defined and analyzed using a Newton Raphson based non-linear solver.

For this specific application it has been chosen to use the (API 2000) definition of the p-y curves for sand,

$p_{u}^{\text {virgin }}(u)=A p_{u l t} \tanh \left(\frac{k X}{A p_{u l t}} u\right)$

Here $p_{u l t}$ is the ultimate capacity, $A$ is a strength reduction parameter, $k$ is the subgrade reaction modulus, $X$ is the depth and $u$ is the total lateral displacement.

Still, in order to implement this relation into the proposed format, it is necessary to divide the total resistance into a drag contribution and a face loading contribution, i.e.

$p_{u}^{\text {virgin }}(u)=p_{u}^{\text {drag }}+p_{u}^{\text {face }}(\boldsymbol{\alpha})$

This is not a trivial task, since the hyperbolic function can not be easily inverted in order to allow 


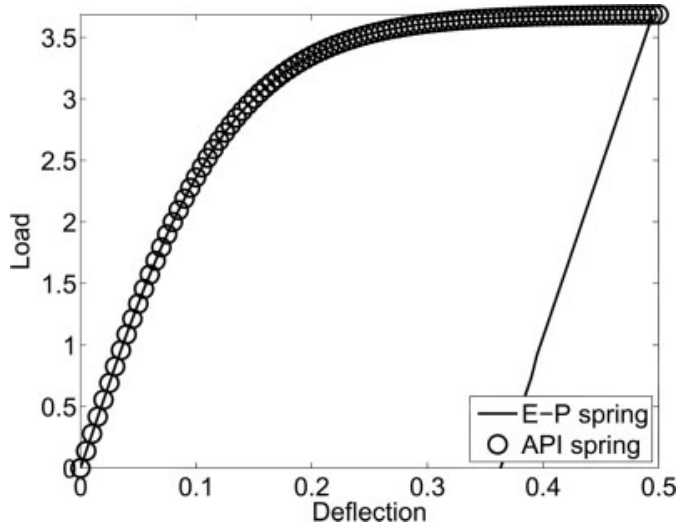

Figure 3. API curve versus the elasto-plastic curve.

Table 1. Pile soil properties.

\begin{tabular}{lll}
\hline Pile diameter & $D$ & $1 \mathrm{~m}$ \\
Pile length & $L$ & $6 \mathrm{~m}$ \\
Load eccentricity & $e$ & $2.5 \mathrm{~m}$ \\
Frictional angle & $\phi$ & $42^{\circ}$ \\
Soil density & $\gamma$ & $16 \mathrm{kN} / \mathrm{m}^{3}$ \\
\hline
\end{tabular}

for a split of elastic and plastic contribution. In the present situation, it has be chosen to use the following approximation,

$p_{u}^{\mathrm{face}}\left(\alpha_{i}\right)=A p_{u l t} \tanh \left(\frac{k X}{A p_{u l t}} u\right)-p_{u}^{\mathrm{drag}}$

Eqn. (17) is a implicit function in $\alpha$ since we have $u=\alpha+p / k$. This implies that the derivative with respect to $\alpha$ is not trivial. Here we use

$\frac{\partial p_{u}}{\partial \alpha} \approx \frac{\partial p_{u}}{\partial u}$

as a first order approximation. Comparing the API curve to the prediction of the model, Figure 3, this approximation appears to be acceptable.

\section{RESULTS}

To demonstrate the ability of the model to capture the pile-soil interaction as observed by Matlock (1970) and Mayoral et al. (2005), three test simulations have been carried out.

The material properties used in the three test examples are shown in Table 1. The three tests have been performed with a monotonic or cyclic laterally load applied in the top of the pile. A rather large stiffness has been used for the sand in order to clearly demonstrate the capability of the spring element.

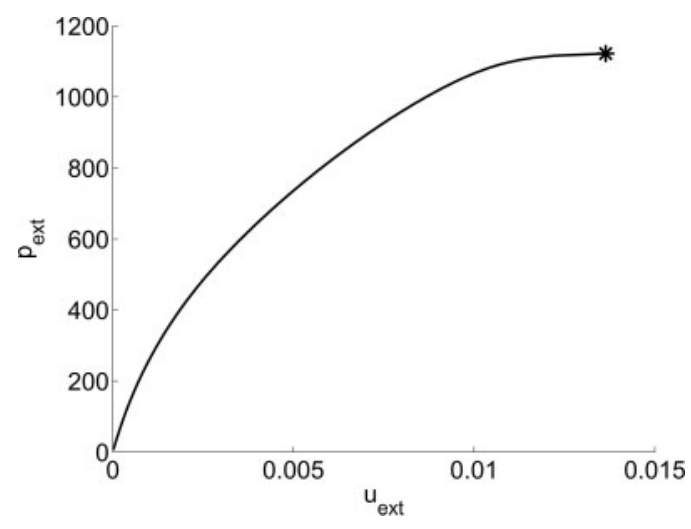

Figure 4. Overall response on a pile subjected to monotonic loading loading.

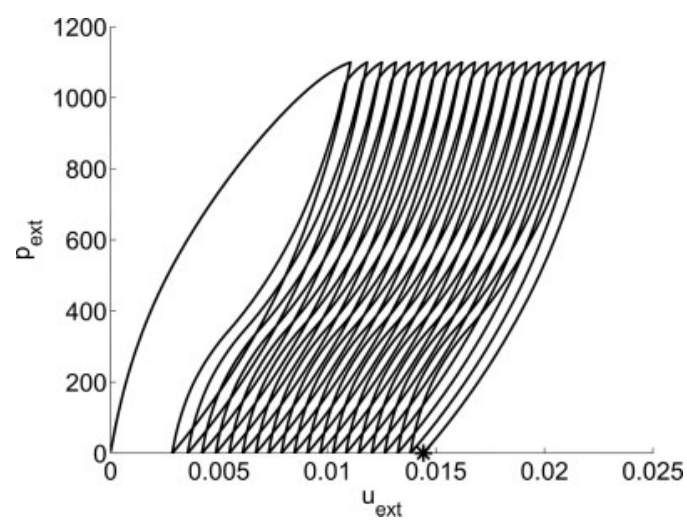

Figure 5. Overall response on a pile subjected to one-way loading.

\subsection{Example 1-monotonic loading}

The spring element presented here is capable of performing cyclic tests. As demonstrated in Figure 3 the elasto-plastic element follows the virgin curve recommended by API (2000). Monotonic tests can therefore also easily be performed with this element. In Figure 4 the result as pile head deflection versus applied laterally load from a monotonic test can be seen. The maximum bearing capacity of the pile is calculated to $P_{\max }=1122 \mathrm{kN}$. Using the theory from Hansen (1961), the maximum bearing capacity can be calculated to $P_{\max }=1152 \mathrm{kN}$. This results fits very well with the calculation performed in the model.

\subsection{Example 2 - one way loading}

The second example illustrates a pile that is subjected to a load varying from zero and to a given value in the same direction, this is called one-way loading. The maximum load during the cycles is close to the ultimate capacity, so that the accumulation effect is clearly seen.

The overall pile response can be seen in Figure 5. This figure shows the pile top deflection versus the 


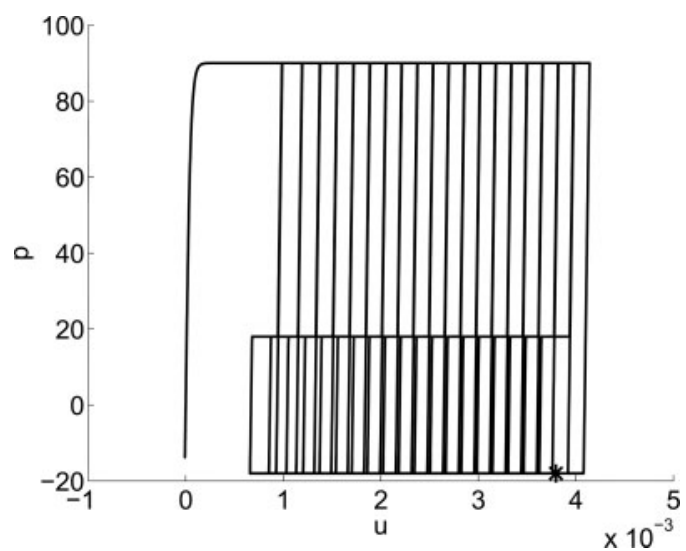

Figure 6. Spring response on a pile subjected to one-way loading.

applied force. The model simulates a load controlled test with constant load amplitude in a total of ten cycles. It can be seen from Figure 5 that the deflection increases with every cycle. Still, the rate of increase for every cycle is getting smaller and smaller. This shows that the model is able to take account for the accumulation of displacement when the model is subjected to one-way loading.

The response from one of the springs near the soil surface can be seen in Figure 6. The spring reaches fast the maximum bearing capacity. This is due to the high stiffness. It unloads elastically and then the development of a cavity can be seen. As described in section 2.2, no hardening occurs when the pile is moving in this cavity. It can also be seen that after the first cycle the the spring does not go back to its initial position, but exhibits a permanent deformation. This is due to the accumulation of deflection. The accumulation of deflections occurs due to the development of cavity in several springs and the subsequent redistribution of the force therefore occurs.

\subsection{Example 3-two way loading}

In this example the pile is subjected to a given load varying between negative and positive values, this is called two-way loading. The overall pile response can be seen in figure Figure 7. The pile is loaded five full cycles. The same maximum force is applied for both direction. It can be seen from Figure 7 that the deflection is getting larger and larger from every load cycle. This is valid for both sides and the increase in deflection is also the same for both sides. This means that the average deflection of the load cycles is constant and equal to zero. It is though interesting that the deflection amplitude increases, hence the secant stiffness will decrease as a consequence of cyclic loading. This effect is extremely important if we are to model the cyclic response of monopile foundations for wind turbine, since the load here is frequency dependent.

It should be noted that the number of iterations increases dramatically after the first half cycle when

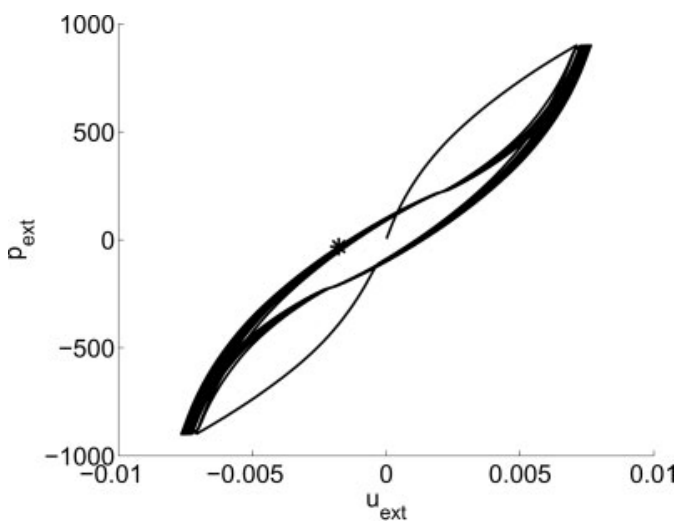

Figure 7. Overall response on a pile subjected to two-way loading.

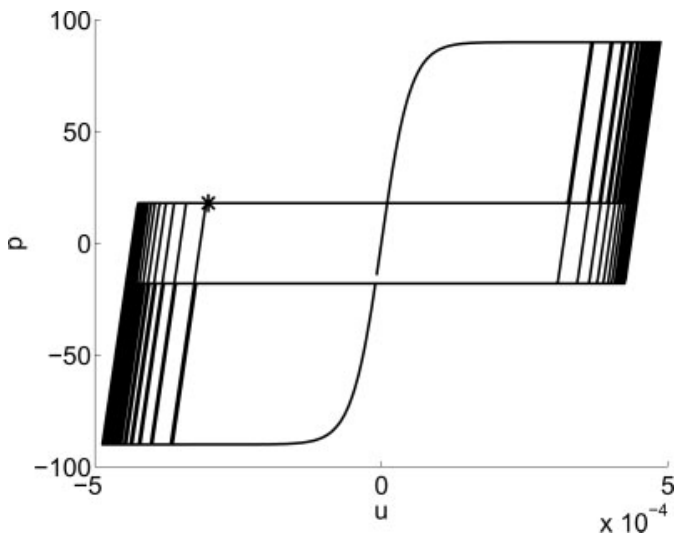

Figure 8. Spring response on a pile subjected to two-way loading.

the pile is in a position around the mean deflection. This is due to the development of a cavity in nearly all spring elements. In this position the system have very low stiffness. A simple remedy to this could be to include a small amount of kinematic hardening to the drag-term in a manner as proposed by Hededal and Strandgaard (2008).

The response from one of the springs can be seen in Figure 8. It can be seen that a cavity develops as expected. As for the overall pile response, an increase in deflection of the single spring for every load cycle is observed. Also here the average deflection for an overall load cycle is constant and equal to zero. There is no degradation of the springs which can be seen in one-way loading example.

\section{DISCUSSION}

The cyclic spring presented in this paper is capable of capture physical aspects as seen in tests Matlock (1970), Mayoral et al. (2005) and Klinkvort (2009). Still, improvements are needed. In this section ideas 
which will improve the performance of the spring element and the representation of the physical world.

The presented model operates with the same virgin stiffness as un-/reloading stiffness. This could be changed and it must also be expected that a soil not will load and unload with the same stiffness. With a change like this the model will probeable start to accumulate displacements in a smaller loading range.

When springs moving in the cavity some sort of hardening should occur. This can also be seen in the Figure 1 by Mayoral et al. (2005). As a side effect an introduction of hardening in the cavity will help the global iterations to converge faster.

Other effects which should be incorporated in the future is suction release for clay springs and the fall back of sand particle when dealing with sand springs.

\section{CONCLUSION}

An elasto-plastic spring element has been defined. The spring element embeds two fundamental features of cyclically loaded piles. It is able to account for preloading of the soil by tracing the virgin curve. Secondly, the creation of a gap after reloading, which is undeniably developing in cohesive soils, is accounted for by introducing a smoothed step function that keeps track of the current position of the pile-soil interfaces. The element is not only relevant for the quasi-static loading with random time series, but also has a potential in dynamic analysis, where it will provide a physically based hysteretic damping.

\section{REFERENCES}

API (2000). American petroleum institute. recommended practice for planning, designing and constructing fixed offshore platforms- working stress design, api recommended practice 2a-wsd (rp2a-wsd), 21st edition, dallas.
Boulanger, R. W., C. J. Curras, B. L. Kutter, D. W. Wilson, and A. Abghari (1999). Seismic soil-pile-structure interaction experiments and analyses. Journal of Geotechnical and Geoenvironmental Engineering 125(9), 750-759.

El-Naggar, M. H., M. A. Shayanfar, M. Kimiaei, and A. A. Aghakouchak (2005). Simplified bnwf model for nonlinear seismic response analysis of offshore piles with nonlinear input ground motion analysis. Canadian Geotechnical Journal 42, 365-380.

Hansen, J. B. (1961). The ultimate resistance of rigid piles against transversal forces. Danish Geotechnical Institute, Copenhagen, Denmark Bulletin NO. 12, 5-9.

Hededal, O. and S. Krenk (1995). FEMLAB - matlab toolbox for the Finite Element Method. Aalborg University.

Hededal, O. and T. Strandgaard (2008). A 3d elasto-plastic soil model for lateral buckling analysis. In Proc. 18th International Offshore and Polar Engineering Conference, ISOPE 2008.

Klinkvort, R. T. (2009). Laterally loaded piles - centrifuge and numerical modelling. Master's thesis, Technical University of Denmark.

Matlock, H. (1970). Correlations for design of laterally loaded piles in soft clay. In Offshore Technology Conference, pp. 577-594.

Matlock, H., H. C. Foo, and L. M. Bryant (1978). Simulation of lateral pile behavior under earthquake motion. In Proc. American Society of Civil Engineers Specialty Conference on Earthquake Engineering and Soil Dynamics, Volume 2, pp. 600-619.

Mayoral, J. M., J. M. Pestana, and R. B. Seed (2005). Determination of multidirectional p-y curves for soft clays. Geotechnical Testing Journal of Computational Mechanics Vol. 28, No.3.

Taciroglu, E., C. Rha, and J. Wallace (2006). A robust macroelement model for soil-pile interaction under cyclic loads. Journal of Geotechnical and Geoenvironmental Engineering, ASCE 132(10), 1304-1314. 